Revue d'histoire de l'Amérique française

REYUE D.HISTOIRE DE L'AMÉRIQUE FRANÇAISE

\title{
Le Musée de la Cathédrale de Moncton
}

Ses souvenirs historiques

\section{Marguerite Michaud}

Volume 8, numéro 2, septembre 1954

URI : https://id.erudit.org/iderudit/301651ar

DOI : https://doi.org/10.7202/301651ar

Aller au sommaire du numéro

Éditeur(s)

Institut d'histoire de l'Amérique française

\section{ISSN}

0035-2357 (imprimé)

1492-1383 (numérique)

Découvrir la revue

\section{Citer cet article}

Michaud, M. (1954). Le Musée de la Cathédrale de Moncton : ses souvenirs historiques. Revue d'histoire de l'Amérique française, 8(2), 236-242.

https://doi.org/10.7202/301651ar d'utilisation que vous pouvez consulter en ligne.

https://apropos.erudit.org/fr/usagers/politique-dutilisation/ 


\section{LE MUSÉE DE LA CATHÉDRALE DE MONCTON}

\section{SES SOUVENIRS HISTORIQUES}

Dans sa largeur d'esprit, le premier Archevêque de Moncton, Son Excellence Mgr L.-J.-A. Melanson, voulait donner essor à tous les mouvements susceptibles de renaissance acadienne. Dès la consécration officielle du "Monument de la Renaissance" (Moncton) en 1940, il jeta la base d'un musée historique dans une des salles du soubassement surnommée la "Salle des Souvenirs".

Son Excellence s'intéressait à l'histoire, à l'éducation, à la vie économique du petit peuple auquel il était fier d'appartenir. Dans un élan littéraire, Mgr Melanson avait conçu le projet d'écrire au courant de l'hiver de 1919-1920 la vie de l'abbé Mathurin Bourg, premier missionnaire d'origine acadienne à exercer son apostolat dans son pays d'origine. (Le missel de l'abbé Bourg, daté de 1740 se trouve parmi les pièces historiques du musée). L'Archevêque de Moncton avait de même écrit l'historique des nouvelles paroisses de St-Quentin (1911) et Kedgwick (1912) dans son "Retour a la Terre"; le crucifix ayant servi lors de la fondation de ses missions a travers ses courses de chantiers occupe une place précieuse parmi les souvenirs personnels de Son Excellence.

Le musée de la Cathédrale est une tranche de l'Histoire de l'Acadie; nombreux sont les ex-voto, les reliques, les documents et registres qui rappellent les diverses étapes de notre histoire. Desireux de conserver ces objets de richesse inestimable, Mgr Melanson s'adressa au peuple, invitant tous ceux qui avaient en leur possession des pièces historiques de bien vouloir les faire parvenir a la Cathedrale afin que l'ensemble demeure comme un hommage vivant au peuple martyr.

Parmi les plus vieux registres se trouve celui de Saint-Louis-deCaraquet (1680) signé de Claude L. Moireau (Récollet, missionnaire). Y sont inscrits les noms des Sauvages des missions de Jemsek, 
Beaubassin, Menagoueche(?). Parmi les noms d'Acadiens qui paraissent dans les mêmes registres, nous remarquons (Beaubassin) 1681, Louise Aucoin, (1682) Pierre Cormier, Jean-Baptiste Poirier; (1683) Cécile Mignaux; (1684) à Rivière-des-Mines, Antoine Pinette, des Hébert, Landry et Melanson. Dans le même registre daté de 1753 et signé de Guerne, nous trouvons les noms de Landry, Babibeau, Bro, Savoye, Melançon, Saunier. (Un excellent article sur l'œuvre de l'abbé Guerne parut dans la Revue d'Histoire l'an dernier, signé du Révérend Père René Baudry). Un peu plus loin est inscrit le mariage de Pierre Luison, soldat du diocèse du Toulouse avec Mlle A. Comeau; ils furent présentés par M. Vergor, commandant $\mathrm{du}$ fort. L'acte est signé par l'abbé LeLoutre. Nous nous rappellerons que ces deux personnages - Le Loutre et Vergor - continuerent la lutte sur l'isthme de Chignectou pour la domination française en Acadie après les événements de 1755.

En date de 1767, est signé le registre de la mission d'Ekopokoy du haut de la Rivière St-Jean, Nouveau-Brunswick; le signataire, l'abbé Charles-François Bailly était acadien par sa mère Marie-Anne Desgouttins, petite-fille du fondateur de Chipoudy, Pierre Thibaudeau. Pendant cinq ans à partir de 1767 , il se dévoua pour les survivants de 1755 dont plusieurs étaient éparpillés le long de la Rivière St-Jean.

Avant de s'occuper des Acadiens de la région de la baie des Chaleurs (1770-1774) le missionnaire Jésuite, Jean-Baptiste de la Brosse, s'était dévoué pour les Acadiens de la région Chignectou. Nous trouvons au musée des registres signés de sa main: "Actes à insérer dans les Registres des paroisses de Pedcokiak et Chepody. Baptême dans la (a) chapelle de Pedkodiak b) ou dans la maison du nommé Toussaint Blanchard où à cause de la guerre je tenais chapelle c) dans une chapelle que les habitants de l'Isle de Petcodiac refugiés aux bois avaient bâtie d) dans une chapelle que les habitants de Chipoudy refugiés aux bois avaient bâtie."

Je soussigné, Prêtre Religieux et missionnaire de la compagnie de Jésus aux Abénakis, Malécites, à la Rivière St-Jean, certifie à tous ceux à qu'il appartient ou appartiendra que par la permission de M. de Guerne, curé de l'Acadie et vicaire général de Monseigneur l'Evêque de Québec, j'ay exerce dans les susdites paroisses les fonctions curiales les 
dessus énoncés conformes à la vérité pour servir comme de raison tant en jugement qu'hors iceluy. Fait à EKK8bak le 16 juin 1756.

Jean-Baptiste de la Brosse, Missionnaire de la Compagnie de Jésus.

Un autre registre de toute première importance pour les Acadiens de la région Kent est consigné au musée; il s'agit des "registres de la région Richibouctou et Kichibouguette, Bouctouche, l'Ardoine, etc." daté du 22 juin 1798 au 30 juillet 1799 et signé des missionnaires L.-J. Desjardins et Joyer (prêtre). L'abbé Desjardins avait parcouru toute la région de la côte occidentale du Nouveau-Brunswick de la baie de Chaleurs jusqu'au détroit de Northumberland.

Quelques précieux volumes de la bibliothèque de l'abbé Manach occupent leur place importante dans la collection historique. Ce missionnaire des "Sauvages de BeauBassin, missionnaire des "Acadiens de Baie des Winds, Richibouctou, Miramichi, etc. etc." était contemporain des Pères Maillard et Germain. Joseph Goguen de Morlaix, France, protégé de l'abbé Manach vint après l'expulsion s'établir à Cocagne en 1768. Il fut gratifié d'une partie de la Bibliothèque Manach. Les Goguen (Gueguen) donnèrent quelques volumes a Marie-Alphonse Erouart qui à son tour les a remis au Révérend J.-A. L'Archevêque, ancien curé de Cocagne. C'est avec plaisir que celui-ci les remit à Son Excellence pour le Musée de la Cathédrale (le 24 aout 1937). Ses qualités d'esprit avaient fait de Joseph Gueguen (Goguen) un vrai missionnaire laïque baptisant les nouveau-nés, célébrant les mariages, disant "la messe blanche". Nombreux sont les documents de famille légués au Père L'Archevêque que celui-ci fit déposer dans le musée de Moncton.

La Mission Ste-Anne est l'ancienne ville de Frédéricton. Tout récemment, le gouvernement fédéral faisait l'acquisition de la résidence autrefois occupée par les gouverneurs de la Province du Nouveau-Brunswick; or, ce site occupe l'emplacement de la vieille chapelle de la Mission détruite par les Hazen Rangers en 1759. Après l'incendie, plusieurs Acadiens se refugièrent dans un village Indien (aujourd'hui nommé Kingsclear à onze milles de la capitale). Quelques-uns y demeurèrent tandis qu'un assez grand nombre filèrent le long de la rivière St-Jean pour ensuite arriver au Madawaska. 
Dans le cimetière de Kingsclear, nous trouvons certains noms français tels que Godin (devenu Goodine) et Mazerolle. Par la suite, quelques-unes de ces familles devinrent protestantes.

Un des vieux registres de l'ancienne Mission Ste-Anne se trouve actuellement au Musée de la Cathédrale.

Le 26 juillet de l'an 1806, nous prêtre curé de St-Basile de Madawaska et missionnaire de la Mission Ste-Anne de Frédéricton avons paragraphé par chaque feuillet pour servir à enregistrer les actes et Baptêmes, Mariages et Sépultures des Catholiques de la susdite mission pendant les années 1806 et suivantes jusqu'à ce que le susdit registre soit rempli.

Charles Hott, prêtre.

A partir du 21 janvier 1811, le registre est signé Ls Baby, prêtre; puis jusqu'au 3 juin 1814, Ls Marcoux, prêtre; 4 juillet 1816, Vital Lefebvre; 14 juin 1818, André Legande; 14 juillet 1822, J.-E. Morifs ( ?). La dernière date est inscrite le 25 juin 1824, Mosifs (?).

En feuilletant cet ancien registre nous trouvons les noms Duplessis, Gaudin, Maserolle, Hébert, Samson, Martin, Roi, Rémond, (soldat du Régiment du Nouveau-Brunswick), Cir, Robichaud, Tanguy (soldat?), Michaud, Dubé, Bourgouin. (Toutes les formes originales de l'orthographe sont conservees.)

Outre les archives décrites, le musée étale les photographies de personnalités acadiennes dans les divers domaines de religion, éducation, politique, histoire, art, journalisme, jurisprudence, lettres, inspectorat. La liste suivante donnera une idée assez succinte des personnes qui ont rendu service soit à l'Acadie même ou à titre d'Acadien.

Placide Gaudet 1850-1930 - grand historien et généalogiste; archiviste à Ottawa.

Le sénateur Pascal Poirier 1852-1933 - premier sénateur acadien; homme de lettres.

Le Père Camille Lefebvre, c.8.c., fondateur de l'Université StJoseph (1864).

Révérend M. F.-X. Lafrance, inspirateur de l'Université St-Joseph, fonda le séminaire Saint-Thomas.

Sir Pierre Landry, juge de la cour Suprême du Nouveau-Brunswick, premier président de l'Assomption Nationale. 
Valentin Landry, inspecteur d'écoles, fondateur du journal l'Evangéline (1887).

Israël Landry, fondateur du journal Le Moniteur.

M. Jérôme Boudreau, (Petit-Rocher) premier inspecteur d'écoles acadien.

Dominique Léger, fondateur de la Caisse Ecolière de la Société l'Assomption (1903).

Gilbert Buote, journaliste de l'Ile-du-Prince-Edouard - L'Impartial.

Gilbert Girouard - député de Kent. Président de la délégation acadienne au congrès de Québec (1880).

Son Honneur le juge Alphonse Turgeon, juge de la cour Suprême de la Saskaltchewan.

Honorable David Landry, ministre d'Agriculture et secrétaire-trésorier provincial. Grand assomptionniste.

Edouard Richard, historien acadien.

Révérend A.-T. Bourque, c.s.c., musicien, auteur des "Chants Acadiens".

Son Excellence Mgr Joseph Léonard, 3e évêqửe de Rimouski.

Mgr Marcel Richard, surnommé père de la Nouvelle Acadie.

Son Excellence Mgr Georges Melançon, 3e évêque de Chicoutimi. Domitien Robichaud, traducteur-en-chef aux Communes d'Ottawa.

Le sénateur Onésiphore Turgeon, auteur de Mémoires.

Son Honneur, le juge Aubin Arseneault de l'Ile du-Prince-Edouard. Père André Cormier, c.s.c., organisateur de pèlerinages à Grand-Pré. Achat de terrain.

Très révérende Mère Léonie, fondatrice des Religieuses de la SainteFamille en Acadie (1878). (Procès de canonisation à Rome actuellement).

S. E. Mgr Blanche, fondateur du Collège Ste-Anne à Church Point, (Pointe de l'Eglise, Nouvelle-Ecosse) 1899.

Mgr L. N. Dugal, P.D., vicaire-général de l'ancien diocèse de Chatham.

Son Excellence Mgr Edouard LeBlanc, premier évêque acadien(1912). Mgr Joseph Melanson, P.D., curé de Berlin, N.H.

Son Exc. Mgr Arthur Beliveau, 3e archevêque de St-Boniface. L'abbé Sylvain Ephrem Poirier, premier prêtre acadien de l'Ile du Prince-Edouard. 
Son Exc. Mgr J.-E. Prince, Evêque de St-Hyacinthe.

Mgr Stanislas Doucet, P.D. de l'ancien diocèse de Chatham. Grand patriote.

Photo de la bénédiction de la pierre angulaire de l'Eglise-Souvenir de Grand.Pré. (Par Son Exc. Mgr Edouard LeBlanc) (1922).

Honorable Charles Labillois, ancien ministre des Travaux Publics au Nouveau-Brunswick. (Dalhousie)

Mgr Théophile Allard, fondateur du Collège du Sacré-Cœur à Cara quet en 1890.

Son Exc. Mgr Patrice Chiasson, premier évêque de Bathurst.

La très révérende Mère Marie de l'Assomption, fondatrice des Filles Marie de l'Assomption à Campbellton en 1924.

La très révérende Mère Marie-Anne, fondatrice des Religieuses Notre-Dme du Sacre-Cœur à St-Joseph en 1924.

Mgr J.-A. Richard, fondateur de la paroisse Notre-Dame des SeptDouleurs à Verdun, près de Montréal.

Mgr Philippe Belliveau, ancien curé de Grand-Digue, Kent, N.-B. $\mathrm{Mgr}$ Henri Cormier, fondateur de la paroisse de l'Assomption de Moncton (1914).

Son Excellence Mgr Albini LeBlanc, 2e évêque de Hearst, évêque actuel de Gaspé.

Son Excellence $M g r$ Norbert Robichaud, archevêqu de Moncton.

Mgr Albert Leménager, premier évêque de Yarmouth, N.-E. (1953). Mgr Alfred Trudel, P.D., Bathurst.

Monsieur H.-H. Melanson, exécutif du Canadien National.

La bénédiction de la pierre angulaire du Monument de la Reconnaissance par son Excellence Mgr L.-J.-A. Melanson, 13 juin 1939.

Les Evangélines en route pour la Louisiane.

Le sénateur T.-J. Bourque de Richibouctou.

M. le docteur Fred Richard, chevalier de l'Ordre de St-Grégoire.

M. le sénateur Antoine Léger, membre de la Société Royale du Canada.

M. le juge Arthur LeBlanc, chevalier de l'ordre de St-Grégoire.

Frère Antoine Bernard, historien de l'Acadie, professeur à l'Université de Montréal.

Mgr Jean Gaudet, chapelain du bataillion acadien 165; curé de Dieppe. Arthur LeBlanc, musicien violoniste. 
Des souvenirs historiques d'une valeur un peu plus personnelle sont compris dans la collection du musée de la Cathédrale. Au nombre de ceux-ci l'on pourrait inclure le chapelet que tenait $\mathrm{Mgr}$ Marcel Richard à sa mort. Ce grand patriote acadien était allé jusqu'à Rome (1910) plaider la cause du clergé acadien en demandant un Evêque de leur race; Sa Sainteté lui avait donné sa promesse - (Mgr Edouard LeBlanc fut sacré Evêque de St-Jean en 1912). Comme gage de sympathie envers le peuple martyr, Pie $X$ lui fit don d'un magnifique chapelet et d'un Calice d'or, cadeau qu'il avait reçu de Dames de l'Écosse lors de son jubilé sacerdotal. Ajoutons au souvenir de Mgr Richard le texte original du décret par lequel Sa Sainteté Pie XI proclamait la Vierge de l'Assomption, patronne liturgique de l'archidiocèse de Moncton, du diocèse de Bathurst, et Patronne céleste de tous les Acadiens (19 janvier 1938).

En évoquant les trésors du musée, le visiteur parcourt tout le trajet du peuple de l'Acadie en voie de renaissance. Il y constate les luttes des premiers missionnaires, les étapes de reconstruction et les témoignages d'une vie nouvelle au XXe siècle. Ce lieu est un symbole vivant de tout ce qui doit être sacré, précieux, historique dans l'âme française du Nouveau-Brunswick.

Marguerite MiChAUD, Professeur au Collège Pédagogique, Fredericton, N.-B. 